Agrotrópica 33(1): 63 - 68. 2021.

Centro de Pesquisas do Cacau, Ilhéus, Bahia, Brasil

\title{
FITORREGULADORES NA RECUPERAÇÃO DA GERMINAÇÃO DE LOTES COMERCIAIS DE SEMENTES DE MARACUJAZEIRO-AZEDO
}

\author{
Adriana Lopes da Luz'1, Jamile da Silva Oliveira², Fábio Gelape Faleiro², Nilton Tadeu Vilela \\ Junqueira ${ }^{2}$, Luis Carlos Galhardo ${ }^{3}$, Welington Fernandes Vieira ${ }^{4}$
}

\begin{abstract}
${ }^{1}$ CNPq. 73310-970, Planaltina - DF, adrianaifg2015@gmail.com; 2Embrapa Cerrados, 73310-970, Planaltina - DF, jamile.oliveira54@gmail.com, fabio.faleiro@embrapa.br,nilton.junqueira@embrapa.br; ${ }^{3}$ Agrocinco, 13190-000,Monte Mor-SP, luis.galhardo@agrocinco.com.br; ${ }^{4}$ Secretaria de Inovação e Negócios, 70770-90, Brasília - DF, welinton.vieira@embrapa.br.
\end{abstract}

Sementes podem se tornar inviáveis ao longo do armazenamento, impossibilitando a comercialização de lotes em razão da baixa capacidade germinativa. Reguladores vegetais em sementes de Passiflora spp. têm sido empregado para potencializar a germinação. Neste trabalho, objetivou-se avaliar o uso de reguladores vegetais na germinação de sementes de lotes comerciais de maracujazeiro-azedo. O delineamento experimental foi inteiramente casualizado em fatorial 8 × 2, com quatro repetições de 50 sementes. Sendo oito lotes de sementes de cultivares com diferentes períodos de armazenamento (BRS RC/18, BRS RC 024/15, BRS RC 028/14, BRS SC/17, BRS SC/16, BRS GA/17, BRS GA/16 e BRS GA/15) e dois tratamentos \{[T1- água destilada e T2- uso de $\mathrm{GA}_{4+7}+\mathrm{N}$-(fenilmetil)- aminopurina (300 ppm)]\}. A germinação foi avaliada aos 7 e aos 28 dias após a incubação. Os dados foram submetidos à análise de variância e as médias dos tratamentos comparadas pelo teste Tukey. Houve efeito significativo dos lotes, dos tratamentos das sementes e também da interação. O tratamento com $\mathrm{GA}_{4+7}+\mathrm{N}$-(fenilmetil) - aminopurina (300 ppm) recuperou a germinação das sementes dos lotes BRS RC 024/15 e BRS RC 028/14, que não seriam mais comercializados por não atender as porcentagens mínimas de germinação exigidas.

Palavras-chave: Passiflora edulis Sims, qualidade fisiológica, armazenamento.

\section{Phytoregulators in the germination recovery of commercial passion fruit seed}

lots. Seeds may become impracticable during storage, making it impossible to sell lots due to the low germination capacity. Vegetable regulators in seeds of Passiflora spp. has been used to enhance germination. In this work, the objective was to evaluate the use of plant regulators in the germination of seeds from commercial batches of passion fruit. The experimental design was completely randomized in a factorial $8 \times 2$, with four replications of 50 seeds. Eight batches of seeds of cultivars with different storage periods (BRS RC / 18, BRS RC 024/15, BRS RC 028/ 14, BRS SC / 17, BRS SC / 16, BRS GA / 17, BRS GA / 16 and BRS GA / 15) and two treatments \{[T1- distilled water and T2- use of $\mathrm{GA}_{4+7}+\mathrm{N}$ - (phenylmethyl) - aminopurine $\left.\left.(300 \mathrm{ppm})\right]\right\}$. Germination was evaluated at 7 and 28 days after incubation. The data were submitted to analysis of variance and the treatment means compared by the Tukey test. There was a significant effect of batches, seed treatments and interaction. The treatment with $\mathrm{GA}_{4+7}+\mathrm{N}-$ (phenylmethyl) - aminopurine (300 ppm) recovered the germination of the seeds of the batches BRS RC 024/15 and BRS RC 028/14, which would no longer be commercialized for not meeting the minimum germination percentages required.

Key words: Passiflora edulis Sims, physiological quality, storage. 


\section{Introdução}

A agricultura é uma das principais bases econômicas do Brasil. As passifloras representam papel fundamental na diversidade de espécies, além de sua grande importância econômica para o país. $\mathrm{O}$ Brasil é o terceiro maior produtor de frutas do mundo, atingindo a produção de aproximadamente 42 milhões de toneladas por ano, e na produção de maracujá, encontra-se como maior produtor e consumidor mundial (554.598 toneladas no ano de 2017) (Anuário Brasileiro de Horti \& Fruti, 2019).

O gênero Passiflora apresenta ampla variabilidade genética, sendo constituído por mais de 500 espécies, dentre as quais, cerca de 150 são originárias do Brasil (Cervi, Milward e Bernacci, 2010). Segundo Meletti e Bruckner (2001), 95\% dos pomares de passifloras no Brasil são formados pelo maracujazeiro-azedo (Passiflora edulis Sims) em decorrência da qualidade dos frutos, vigor, produtividade e rendimento em suco proporcionados pela espécie.

A propagação do maracujazeiro pode ocorrer de forma sexuada através das sementes ou assexuada pelos métodos de estaquia enxertia ou cultura de tecidos. No entanto, a propagação por sementes dispõe de alta variabilidade e baixa uniformidade para as plantas (Grattaplagia et al., 1991; Alexandre et al., 2004; Lima e Trindade, 2004; Faleiro et al., 2019).

Alguns estudos afirmam que a germinação de sementes do gênero Passiflora ocorrem de forma irregular variando entre dez dias e três meses, o que interfere na produção de mudas de qualidade pela falta de uniformização, além disso as sementes podem perder sua viabilidade ao longo do tempo de armazenamento (Pereira e Dias, 2000).

Fitormônios são substâncias naturais desenvolvidas pelas plantas, e os termos regulador de crescimento ou regulador de crescimento vegetal são utilizados para substâncias naturais ou artificiais que proporcionam efeitos no crescimento e desenvolvimento de vegetais (Melo, 2002). O desenvolvimento de compostos naturais ou sintéticos tem demonstrado bons resultados na agricultura, em especial para a fruticultura, já que, estes compostos possibilitam o aumento de áreas de cultivo de fruteiras de modo que pode vir a influenciar, manter ou aumentar a qualidade, a produtividade, a colheita e etc. (Petri et al., 2016).

Sendo assim, vale ressaltar a importância dos estudos que buscam reconhecer os aspectos e fatores que afetam negativamente a germinação de sementes, tais como fatores ambientais, fatores genéticos, fatores de pré e pós-colheita (danos mecânicos relacionados à colheita do fruto, secagem e armazenamento) danos morfológicos e fisiológicos como dormência, maturidade, vigor, dentre outros (Pádua et al., 2011).

Avaliações acerca da capacidade germinativa das sementes em decorrência de diferentes condições são essenciais para traçar estratégias de armazenamento e para desenvolver processos comerciais de produção de mudas. Neste trabalho, objetivou-se avaliar a germinação de sementes de lotes comerciais de maracujazeiro-azedo (Passiflora edulis Sims) e analisar o efeito do uso de reguladores vegetais na recuperação da germinação das sementes.

\section{Material e Métodos}

O estudo foi realizado no Laboratório de Genética e Biologia Molecular da Embrapa Cerrados em Planaltina-DF, no período de maio à outubro de 2019, utilizando-se sementes de três cultivares comerciais de maracujazeiro-azedo (BRS Rubi do Cerrado, BRS Sol do Cerrado e BRS Gigante Amarelo). As sementes utilizadas no experimento foram oriundas da Agrocinco, empresa licenciada pela Embrapa para produção e comercialização de sementes.

Para cada lote, foi montado um experimento em Delineamento Inteiramente Casualizado (DIC), em esquema fatorial $8 \times 2$, sendo oito lotes de sementes com diferentes períodos de armazenamento (BRS RC/ 18 - 1 ano, BRS RC 024/15 - 4 anos, BRS RC 028/14 - 5 anos, BRS SC/17 - 2 anos, BRS SC/16 - 3 anos, BRS GA/17 - 2 anos, BRS GA/16 - 3 anos e BRS GA/15 - 4 anos) e dois tratamentos de sementes, sendo: $\left\{\left[\mathrm{T} 1\right.\right.$ - água destilada e T2- uso de $\mathrm{GA}_{4+7}+\mathrm{N}-$ (fenilmetil) - aminopurina (300 ppm)]\}, com quatro repetições totalizando 64 parcelas experimentais, sendo cada parcela experimental composta por 50 sementes.

As sementes de cada lote foram embebidas pelo período de 30 minutos em ambos os tratamentos 
(T1 e T2). Após o tempo de imersão as mesmas foram distribuídas em papel de germinação umedecido com água destilada na quantidade correspondente a 2,5 vezes a massa do papel não hidratado, os rolos de papel foram acondicionados em câmara de germinação à $25^{\circ} \mathrm{C}$ noturno e $30^{\circ} \mathrm{C}$ diurno, fotoperíodo 11 horas de luz. A análise e contagem do número de sementes germinadas foi realizada aos 7 e aos 28 dias após a incubação, conforme estabelecido pelas Regras Para Análise de Sementes, RAS (Brasil, 2009).

Para atender as pressuposições da homogeneidade de variância e distribuição normal dos erros, os dados obtidos foram submetidos à transformação arc sen $\sqrt{ }(\mathrm{X} / 100)$ e posteriormente submetidos à análise de variância e as médias dos tratamentos foram comparadas pelo teste de Tukey a $1 \%$ de significância utilizando o programa Genes (Cruz, 2013).

\section{Resultados e Discussão}

Observou-se através da análise de variância que houve efeito altamente significativo tanto do tratamento com $\mathrm{GA}_{4+7}+\mathrm{N}$-(fenilmetil) aminopurina (300 ppm) quanto dos lotes em ambas as contagens de germinação (aos 7 e aos 28 dias). Também houve efeito altamente significativo da interação tratamento $\mathrm{x}$ lote demonstrando que o efeito positivo do promotor de germinação depende do lote de sementes analisado (Tabela 1).
A maior diferença entre as porcentagens de germinação foi observada na primeira contagem aos sete dias, indicando que o tratamento de sementes com regulador vegetal promoveu expressão do potencial germinativo das sementes, com exceção do lote GA/17 que apresentou porcentagem de germinação baixa para os dois tratamentos (Tabela 2). Tais resultados confirmam que os tratamentos com giberelina e citocinina de modo isolado ou em misturas melhoram significativamente a germinação de sementes (Ferreira, 1998).

Na Tabela 3, observa-se que aos 28 dias após a incubação, as sementes dos lotes RC/18, SC/16, GA/16 e GA/15 apresentaram elevados valores de germinação (acima de 95\%) para os dois tipos de tratamentos, indicando que, em lotes com maiores porcentagens de germinação não há necessidade de tratamento com promotor de germinação (Tabela 3).

Os lotes de RC/024/15 e RC/028/14 apresentaram maiores porcentagens de germinação quando foi feito o tratamento com reguladores vegetais. Este tratamento propiciou um aumento da porcentagem de germinação de $53,5 \%$ para $81 \%$ (lote RC/024/15) e de $70,5 \%$ para $82,5 \%$ (lote RC/028/14). Estes são os dois lotes com maior tempo de armazenamento com 4 e 5 anos respectivamente (Tabela 3). Sendo assim, o uso de reguladores vegetais foi crucial para elevar o potencial germinativo, viabilizando a sua comercialização em acordo com os padrões estabelecidos pela a Instrução Normativa ${ }^{\circ}$ 42/2019 (Espécies olerícolas, condimentares, medicinais e

Tabela 1. Resumo da análise de variância de oito lotes de maracujazeiro-azedo para as variáveis primeira contagem da germinação (\%, sete dias), segunda contagem da germinação (\%, 28 dias) e porcentagem total da germinação. Embrapa Cerrados, Planaltina, DF, 2019

\begin{tabular}{|c|c|c|c|c|c|c|c|}
\hline \multirow{2}{*}{ FV } & \multirow{2}{*}{ GL } & \multicolumn{2}{|c|}{$1^{\mathrm{a}}$ Contagem } & \multicolumn{2}{|c|}{$2^{a}$ Contagem } & \multicolumn{2}{|c|}{ Germinação Total } \\
\hline & & QM & $\mathbf{F}$ & QM & $\mathbf{F}$ & QM & $\mathbf{F}$ \\
\hline Tratamento & 1 & 41056,89 & $830,39^{* *}$ & 34086,39 & $1016,08^{* *}$ & 324,00 & $8,55^{*}$ \\
\hline Lote & 7 & 1208,64 & $24,44^{* *}$ & 1600,48 & $47,71^{* *}$ & 4565,05 & $120,54^{* *}$ \\
\hline Tratamento*Lote & 7 & 649,60 & $13,30^{* *}$ & 889,75 & $26,52^{* *}$ & 212,43 & $5,61^{* *}$ \\
\hline Resíduo & 48 & 49,44 & & 33,54 & & 37,87 & \\
\hline \multirow{2}{*}{\multicolumn{2}{|c|}{$\begin{array}{l}\text { MG } \\
\mathrm{CV}(\%)\end{array}$}} & \multirow{2}{*}{\multicolumn{2}{|c|}{$\begin{array}{l}44,45 \\
11,44\end{array}$}} & \multicolumn{2}{|c|}{37,11} & \multicolumn{2}{|c|}{81,56} \\
\hline & & & & \multicolumn{2}{|c|}{10,55} & \multicolumn{2}{|c|}{7,29} \\
\hline
\end{tabular}

FV - fonte de variação, GL - grau de liberdade, QM - quadrado médio, F - de Fisher, MG - média geral, CV - coeficiente de variação. ${ }^{*}$ Significativo a $5 \%$ de probabilidade de erro $(\mathrm{p}>0,01$ a 0,05$),{ }^{* *}$ Altamente significativo $(\mathrm{p}<0,001)$. 
Tabela 2. Médias da interação entre os tratamentos e os lotes de sementes das cultivares de maracujazeiro-azedo para a primeira contagem da germinação (\%, sete dias). Embrapa Cerrados, Planaltina, DF, 2019

\begin{tabular}{lllllllll}
\hline $\mathbf{T} / \mathbf{L}$ & $\mathbf{R C} / \mathbf{1 8}$ & $\mathbf{R C} / \mathbf{0 2 4} / \mathbf{1 5}$ & $\mathbf{R C} / \mathbf{0 2 8} / \mathbf{1 4}$ & $\mathbf{S C} / \mathbf{1 7}$ & $\mathbf{S C} / \mathbf{1 6}$ & $\mathbf{G A} / \mathbf{1 7}$ & $\mathbf{G A} / \mathbf{1 6}$ & $\mathbf{G A} / \mathbf{1 5}$ \\
\hline $\mathrm{SP}$ & $27,00 \mathrm{Ab}$ & $22,75 \mathrm{Ab}$ & $24,25 \mathrm{Ab}$ & $14,50 \mathrm{Ab}$ & $18,00 \mathrm{Ab}$ & $10,50 \mathrm{Aa}$ & $20,00 \mathrm{Ab}$ & $16,00 \mathrm{Ab}$ \\
$\mathrm{CP}$ & $83,00 \mathrm{Aa}$ & $74,00 \mathrm{Aa}$ & $70,75 \mathrm{Aa}$ & $78,00 \mathrm{Aa}$ & $78,50 \mathrm{Aa}$ & $19,00 \mathrm{Ba}$ & $75,50 \mathrm{Aa}$ & $79,50 \mathrm{Aa}$ \\
\hline
\end{tabular}

As médias seguidas pela mesma letra maiúscula na linha e minúscula na coluna não diferem estatisticamente entre si pelo teste de Tukey a $1 \%$ de probabilidade de erro.

Legenda: T - Tratamento de sementes ([SP- água destilada e CP- uso do regulador vegetal GA4+7 + N-(fenilmetil)- aminopurina $\left(15 \mathrm{ml} \mathrm{L}^{-1}\right)$ ]), L - lotes (BRS Rubi do Cerrado - RC/18, BRS Rubi do Cerrado - RC/024/15, BRS Rubi do Cerrado - RC/028/14, BRS Sol do Cerrado - SC/17, BRS Sol do Cerrado - SC/16, BRS Gigante Amarelo - GA/17, BRS Gigante amarelo - GA/16 e BRS Gigante Amarelo - GA/15).

Tabela 3. Médias da interação entre os tratamentos e os lotes de sementes das cultivares de maracujazeiro-azedo para a porcentagem total de germinação (28 dias), Embrapa Cerrados, Planaltina, DF, 2019

\begin{tabular}{lcccccccc}
\hline T/L & $\mathbf{R C} / \mathbf{1 8}$ & $\mathbf{R C} / \mathbf{0 2 4} / \mathbf{1 5}$ & $\mathbf{R C} / \mathbf{0 2 8} / \mathbf{1 4}$ & $\mathbf{S C} / \mathbf{1 7}$ & $\mathbf{S C} / \mathbf{1 6}$ & $\mathbf{G A} / \mathbf{1 7}$ & $\mathbf{G A} / \mathbf{1 6}$ & $\mathbf{G A} / \mathbf{1 5}$ \\
\hline $\mathrm{SP}$ & $99,00 \mathrm{Aa}$ & $53,50 \mathrm{Cb}$ & $70,50 \mathrm{Cb}$ & $89,50 \mathrm{Ba}$ & $97,50 \mathrm{ABa}$ & $30,00 \mathrm{Da}$ & $97,50 \mathrm{ABa}$ & $97,00 \mathrm{Aba}$ \\
$\mathrm{CP}$ & $99,00 \mathrm{Aa}$ & $81,00 \mathrm{Ca}$ & $82,50 \mathrm{Ca}$ & $88,00 \mathrm{BCa}$ & $98,00 \mathrm{ABa}$ & $29,50 \mathrm{Da}$ & $97,00 \mathrm{ABa}$ & $95,50 \mathrm{ABCa}$ \\
\hline
\end{tabular}

As médias seguidas pela mesma letra maiúscula na linha e minúscula na coluna não diferem estatisticamente entre si pelo teste de Tukey a $1 \%$ de probabilidade de erro.

Legenda: T - Tratamento de sementes ([SP-água destilada e CP- uso do regulador vegetal GA4+7 + N-(fenilmetil)- aminopurina (15 ml L $\left.\left.\mathrm{L}^{-1}\right)\right]$ ), L - lotes (BRS Rubi do Cerrado - RC/18, BRS Rubi do Cerrado - RC/024/15, BRS Rubi do Cerrado - RC/028/14, BRS Sol do Cerrado - SC/17, BRS Sol do Cerrado - SC/16, BRS Gigante Amarelo - GA/17, BRS Gigante amarelo - GA/16 e BRS Gigante Amarelo - GA/15).

aromáticas) que determina a germinação mínima para Passiflora edulis Sims de $75 \%$ para que as sementes sejam comercializadas.

As sementes do lote GA/17 apresentaram uma baixa porcentagem de germinação aos 28 dias, ficando abaixo da recomendada para comercialização. Neste caso, o tratamento de sementes com reguladores vegetais não melhorou a porcentagem de germinação. Apesar de não ter sido comprovado, pressupõe-se que este lote tenha sofrido algum dano no momento de sua obtenção (beneficiamento das sementes) ou alguma inadequação nas condições de armazenamento das sementes. Segundo Moore (1974), em processos de produção mecanizada de sementes, danos mecânicos representam os maiores problemas que operam sob a redução da qualidade fisiológica e sanitária dessas (Tabela 3).

Os resultados obtidos no presente estudo são positivos especialmente do ponto de vista da comercialização das sementes das cultivares tendo em vista que a utilização do regulador vegetal promoveu o aumento das porcentagens de germinação e a aceleração do processo germinativo, o que favorece a formação de mudas por uniformizar a germinação e diminuir o tempo de exposição aos patógenos. Segundo Carvalho et al., 2018, um dos maiores impasses enfrentados na comercialização de sementes está na perda de vigor e viabilidade de sementes armazenadas, e conforme os resultados obtidos no presente trabalho, foi possível observar que o uso de reguladores vegetais promoveu a recuperação de lotes que estavam inviáveis para a comercialização.

\section{Conclusão}

A utilização de reguladores vegetais $\mathrm{GA}_{4+7}+\mathrm{N}$ (fenilmetil) - aminopurina (300 ppm) no tratamento de sementes de maracujazeiro-azedo demonstraram uma melhora na germinação, além de potencializar ou recuperar o processo de germinação de sementes em lotes armazenados por longos períodos, recuperando em alguns casos, as exigências mínimas de comercialização. 
A utilização dos reguladores vegetais $\mathrm{GA}_{4+7}+\mathrm{N}-$ (fenilmetil) - aminopurina (300 ppm) no tratamento de sementes de maracujazeiro-azedo, promoveu o aumento das porcentagens de germinação e a aceleração do processo germinativo, em lotes de sementes armazenados por longos períodos, recuperando em alguns casos, as exigências mínimas de comercialização.

\section{Literatura Citada}

ALEXANDRE, R. S. et al. 2004. Germinação de sementes de genótipos de maracujazeiro. Pesquisa Agropecuária Brasileira 39(12):12391245.

ANUÁRIO BRASILEIRO DE HORTI \& FRUTI. 2019. Benno Bernardo Kist. [et al.]. - Santa Cruz do Sul: Editora Gazeta Santa Cruz, 2018.96p.: il. Disponível em: http://www.editoragazeta.com.br/ sitewp/wpcontent/uploads/2019/07/ HortiFruti_2019_DUPLA.pdf $>$ Acesso em:15 nov. 2019.

BRASIL. MINISTÉRIO DA AGRICULTURA, PECUÁRIA E ABASTECIMENTO. 2009. Regras para análise de sementes. Secretaria de Defesa Agropecuária. Brasília, DF, Mapa/ACS. 399 p.

CARVALHO, R. V.; FALEIRO, F. G.; OLIVEIRA, J. S.; JUNQUEIRA, N. T. V.; COSTA, A. M.; PÁDUA, J. G.; MALAQUIAS, J. V. 2018. Armazenamento de sementes de Passiflora alata, Passiflora cincinnata e Passiflora setacea em embalagens aluminizadas à temperatura ambiente. Magistra (Brasil) 29(2):154-160.

CERVI, A. C.; MILWARD, A.; BERNACCI, L. C. 2010. Passifloraceae. In: Lista de Espécies da Flora do Brasil. Jardim Botânico do Rio de Janeiro. Disponível em: $<$ http://floradobrasil. jbrj.gov.br/ 2010/FB000182> Acesso em:15 nov. 2019.

CRUZ, C. D. 2013. GENES - a software package for analysis in experimental statistics and quantitative genetics. Acta
Scientiarum Agronomy (Brasil) 35(3):271276.

FALEIRO, F. G. et al. 2019. Advances in passion fruit propagation. Revista Brasileira de Fruticultura 41(2):e-155.

FERREIRA, G. 1998. Estudo da embebição e do efeito de fitorreguladores na germinação de sementes de Passifloráceas. Tese Doutorado. Faculdade de Ciências Agronômicas, Universidade Estadual Paulista, Botucatu, SP.

GRATTAPAGLIA, D. et al. 1991. Cultura de tecidos de maracujá. In: São José, A. R. (ed.) A cultura do maracujá no Brasil. Jaboticabal, SP, FUNEP. pp.61-77.

LIMA, A. A.; TRINDADE, A. V. 2004. Maracujá: Produção e Qualidade na Passiflora. Embrapa Mandioca e Fruticultura, Cruz das Almas, BA. pp.109-116.

MELETTI, L. M. M.; BRÜCKNER, C. H. 2001. Melhoramento Genético. In: Brückner, C.H.; Picanço, M.C. Maracujá: tecnologia de produção, pós-colheita, agroindústria, mercado. Porto Alegre, SC, Cinco Continentes. pp.345-385.

MELO, F. N. 2002. Hormônios e reguladores de crescimento vegetal. In: Seminário Coda de Nutrição Vegetal, 1. Embrapa semi-árido, Brasilia, DF. Disponível em:< file://C:/Users/Adriana/ Downloads/Hormonios-e-Reguladores-deCrescimento-Vegetal.pdf $>$ Acesso em:27 out. 2019.

MOORE, R. P. 1974. Effects of mechanical injuries on viability. In: E. H. Roberts, (ed.). Viability of seeds. London: Chapman and Hall. 448p.

PÁDUA, J. G.; SCHWINGEL, L. C.; MUNDIM, R. C.; SALOMÃO, A. N.; ROVERIJOSÉ, S. C. B. 2011. Germinação de sementes de Passiflora setacea e dormência induzida pelo armazenamento. Revista Brasileira de Sementes, Brasília, 33(1):80-85.

PEREIRA, K. J. C.; DIAS, D. C. F. 2000. Germinação e vigor de sementes de maracujá-amarelo 
(Passiflora edulis Sims f. flavicarpa Deg.) submetidas a diferentes métodos de remoção da mucilagem. Revista Brasileira de Sementes 22:288-291.
PETRI, J. L. et al. 2016. Reguladores de crescimento para frutíferas de clima temperado. Florianópolis, SC, Epagri. 141p. 